\title{
Optimization of siRNA Delivery Method into the Liver by Sequential Injection of Polyglutamic Acid and Cationic Lipoplex
}

\author{
Yoshiyuki Hattori*, Shohei Arai, Takuto Kikuchi, Megumi Hamada, Ryou Okamoto, \\ Yoko Machida, Kumi Kawano \\ Department of Drug Delivery Research, Hoshi University, Tokyo, Japan \\ Email: ${ }^{*}$ hhattori@hoshi.ac.jp
}

Received 23 April 2015; accepted 17 July 2015; published 22 July 2015

Copyright (C) 2015 by authors and Scientific Research Publishing Inc.

This work is licensed under the Creative Commons Attribution International License (CC BY). http://creativecommons.org/licenses/by/4.0/

\section{(c) (i) Open Access}

\begin{abstract}
Previously, we developed a novel siRNA transfer method to the liver by sequential intravenous injection of poly-L-glutamic acid (PGA) and cationic liposome/siRNA complex (cationic lipoplex). In this study, we examined the effects of the charge ratio (+/-) of cationic liposome/siRNA, molecular weight of PGA and cationic lipid of cationic liposome on the biodistribution of siRNA after sequential injection of PGA plus cationic lipoplex. When 1,2-dioleoyl-3-trimethylammonium-propane (DOTAP)/cholesterol (Chol) lipoplex was intravenously injected into mice, the accumulation of siRNA was mainly observed in the lungs. In contrast, when DOTAP/Chol lipoplex was intravenously injected at 1 min after intravenous injection of PGA, siRNA was largely accumulated in the liver. The charge ratio (+/-) of DOTAP/Chol liposome/siRNA did not affect the biodistribution of siRNA after sequential injection. As regards the molecular weight of PGA, the accumulation of siRNA was observed mainly in the liver after the sequential injection of PGA of 20.5, 38, 64 or 200 kDa plus DOTAP/Chol lipoplex. Furthermore, to examine the effect of cationic lipid of cationic liposome on the biodistribution of siRNA, we prepared other cationic liposomes composed of 1,2-di$O$-octadecenyl-3-trimethylammonium propane chloride (DOTMA)/Chol, dimethyldioctadecylammonium bromide (DDAB)/Chol and $O, O^{\prime}$-ditetradecanoyl- $N$ - $(\alpha$-trimethylammonioacetyl)di-ethanolamine chloride (DC-6-14)/Chol. For the cationic liposomes, the accumulation of siRNA was observed mainly in the liver when their cationic lipoplexes were sequentially injected after injection of PGA into mice. From these findings, sequential injection of PGA plus cationic lipoplex could deliver siRNA efficiently into the liver regardless of the charge ratio (+/-) of lipoplex, lengths of PGA and cationic lipid of liposome.
\end{abstract}

"Corresponding author.

How to cite this paper: Hattori, Y., Arai, S., Kikuchi, T., Hamada, M., Okamoto, R., Machida, Y. and Kawano, K. (2015) Optimization of siRNA Delivery Method into the Liver by Sequential Injection of Polyglutamic Acid and Cationic Lipoplex. Pharmacology \& Pharmacy, 6, 302-310. http://dx.doi.org/10.4236/pp.2015.67032 


\section{Keywords}

\section{Cationic Liposome, siRNA Delivery, Polyglutamic Acid, Liver Targeting, Sequential Injection}

\section{Introduction}

RNA interference (RNAi) is a powerful gene-silencing process that holds great promise in the field of gene therapy [1]. The liver is an important organ with a number of potential therapeutic siRNA targets, including cholesterol biosynthesis, fibrosis, hepatitis and hepatocellular carcinoma. Although none of the currently available methods of siRNA delivery is optimal for liver siRNA therapy, concerted effort from researchers has provided a wide range of choices for siRNA transfer to the liver. Generally, siRNAs fail to cross biological membranes by passive diffusion owing to their high molecular weight and anionic nature. Therefore, carriers of siRNA are required to deliver siRNA into the cytoplasm of target cells. In siRNA delivery, non-viral vectors such as cationic liposomes and cationic polymers have been more commonly used than viral vectors [2] [3]. Among all of the carriers, lipid-based formulations such as cationic liposomes are currently the most widely validated means for the systemic delivery of siRNA to the liver [4].

For efficient siRNA delivery to the liver by cationic liposomes, the cationic liposome/siRNA complex (cationic lipoplex) must be stabilized in the blood by avoiding its agglutination with blood components, and the pharmacokinetics of lipoplex after intravenous injection must be controlled because electrostatic interactions between positively charged lipoplexes and negatively charged erythrocytes cause agglutination [5], and the agglutinates contribute to high entrapment of lipoplex in the highly extended lung capillaries [6].

Poly-L-glutamic acid (PGA) is a polyamino acid having one carboxylic acid per glutamic acid unit. Previously, we developed PGA-coated lipoplex of siRNA (ternary complex of PGA, siRNA and cationic liposomes) and found that PGA coatings for cationic lipoplex prevented the accumulation of lipoplex in the lungs by inhibiting interaction with erythrocytes and could deliver siRNA to the liver [7]. Thereafter, we found that pre-treatment of erythrocytes with PGA could prevent agglutination in vitro after addition of cationic lipoplex [8]. Therefore, we revisited a previously reported gene transfer method [7] and developed sequential injection of PGA and cationic lipoplex for systemic gene delivery of siRNA into the liver efficiently without accumulation in the lung [8]. However, it was not clear whether siRNA delivery into the liver by sequential injection of PGA plus cationic lipoplex was affected by the molecular weight of PGA, the charge ratio (+/-) of cationic liposome/siRNA and the cationic lipid of cationic liposome. Therefore, in this study, we examined the optimal conditions of siRNA delivery into the liver by sequential injection of PGA and cationic lipoplex.

\section{Materials and Methods}

\subsection{Materials}

1,2-Dioleoyl-3-trimethylammonium-propane methyl sulfate salt (DOTAP) was obtained from Avanti Polar Lipids Inc. (Alabaster, AL, USA). 1,2-Di-O-octadecenyl-3-trimethylammonium propane chloride (DOTMA) was purchased from Tokyo Kasei Co., Ltd. (Tokyo, Japan). Dimethyldioctadecylammonium bromide (DDAB, product name DC-1-18) and $O, O^{\prime}$-ditetradecanoyl- $N$-( $\alpha$-trimethylammonioacetyl)diethanolamine chloride (DC6-14) were obtained from Sogo Pharmaceutical Co., Ltd. (Tokyo, Japan). Poly- $\alpha$-L-glutamic acid sodium salt (PGA, $20.5 \mathrm{kDa}$ ), poly- $\alpha$-L-glutamic acid sodium salt (64 kDa) and poly- $\alpha$-D-glutamic acid sodium salt (38 kDa) were purchased from Sigma-Aldrich Co. (St. Louis, MO, USA). Poly- $\gamma$-L-glutamic acid sodium salt (200 kDa) and cholesterol (Chol) were purchased from Wako Pure Chemical Industries, Ltd. (Osaka, Japan). All other chemicals were of the finest grade available.

\subsection{SIRNA}

Firefly pGL3 luciferase siRNA (siRNA) and Cy5.5-labeled Luc siRNA (Cy5.5-siRNA) were synthesized by Sigma Genosys (Tokyo, Japan). The siRNA sequences of the pGL3 luciferase siRNA were as follows: sense strand: 5'-GUGGAUUUCGAGUCGUCUUAA-3', and antisense strand: 5'-AAGACGACUCGAAAUCCACAU-3'. In Cy5.5-siRNA, Cy5.5 dye was conjugated at the 5'-end of the sense strand. 


\subsection{Preparation of Liposome and Lipoplex}

For the preparation of cationic liposomes, we used four kinds of cationic lipid, DOTAP, DOTMA, DDAB and DC-6-14 (Figure 1). Cationic liposomes were prepared from DOTAP/Chol, DOTMA/Chol, DDAB/Chol and DC-6-14/Chol at a molar ratio of $1 / 1$ by a thin-film hydration method, as previously reported [7]. To prepare cationic liposome/siRNA complex (cationic lipoplex), cationic liposome suspension was mixed with siRNA by vortexing for $10 \mathrm{~s}$ at charge ratios (+/-) of 4/1, 6/1 and 8/1, and left for $15 \mathrm{~min}$ at room temperature. The theoretical charge ratio (+/-) of cationic liposome to siRNA was calculated as the molar ratio of DOTAP, DOTMA, DDAB or DC-6-14 nitrogen to siRNA phosphate.

The particle size distributions of lipoplex were measured by the cumulant method using a light-scattering photometer (ELS-Z2, Otsuka Electronics Co., Ltd., Osaka, Japan) at $25^{\circ} \mathrm{C}$, after diluting the dispersion to an appropriate volume with water. The $\zeta$-potentials were measured by electrophoresis light-scattering methods using ELS-Z2 at $25^{\circ} \mathrm{C}$, after diluting the dispersion to an appropriate volume with water. When DOTAP, DOTMA, DDAB and DC-6-14 liposomes were mixed with siRNA at a charge ratio (+/-) of 4/1, their lipoplex sizes were about $340 \mathrm{~nm}, 203 \mathrm{~nm}, 418 \mathrm{~nm}$ and $307 \mathrm{~nm}$, and their $\zeta$-potentials were about $41 \mathrm{mV}, 40 \mathrm{mV}, 43 \mathrm{mV}$ and 34 $\mathrm{mV}$, respectively.

\subsection{Biodistribution of siRNA After Intravenous Injection of Cationic Lipoplex to Mice}

All animal experiments were performed with approval from the Institutional Animal Care and Use Committee of Hoshi University. At $1 \mathrm{~min}$ after intravenous injection of solution of $1 \mathrm{mg}$ of PGA, cationic lipoplexes of $50 \mu \mathrm{g}$ of Cy5.5-siRNA were intravenously administered via lateral tail veins into female BALB/c mice (8 weeks of age; Sankyo Lab. Service Corp., Tokyo, Japan). One hour after the injection of cationic lipoplex, the mice were sacrificed, and Cy5.5 fluorescent imaging of the tissues was performed using a NightOWL LB981 NC100 system (Berthold Technologies, Bad Wildbad, Germany). The excitation and emission filters were set at 630/20 and $680 / 30 \mathrm{~nm}$, respectively. The exposure time for fluorescence was 5 seconds. A grayscale body-surface reference image was collected using a NightOWL LB981 CCD camera. The images were analyzed using the IndiGo2 software provided with the in vivo imaging system (Berthold Technologies). The tissues after fluorescent imaging were frozen on dry ice and sliced at $16 \mu \mathrm{m}$. The localization of Cy5.5-siRNA was examined using an Eclipse TS100-F microscope (Nikon, Tokyo, Japan).
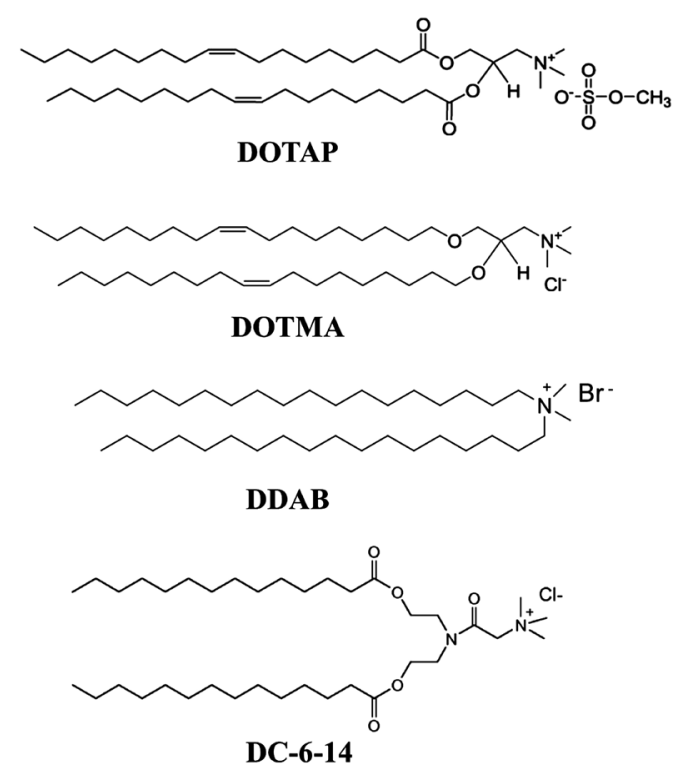

Figure 1. Chemical structure of cationic lipids for preparation of cationic liposome. 1,2-Dioleoyl-3-trimethylammonium propane methyl sulfate, DOTAP; 1,2-di-O-octadecenyl-3-trimethylammonium propane chloride, DOTMA; dimethyldioctadecylammonium bromide, DDAB; $O, O^{\prime}$-ditetradecanoyl- $N$ - $(\alpha$-trimethylammonioacetyl)diethanolamine chloride, DC-6-14. 


\subsection{Agglutination Assay}

Agglutination assay was performed according to the method described in a previous report [7]. Briefly, erythrocytes were collected from mouse blood at $4^{\circ} \mathrm{C}$ by centrifugation at $300 \mathrm{~g}$ for $3 \mathrm{~min}$ and resuspended in PBS as a $2 \%(\mathrm{v} / \mathrm{v})$ stock suspension of erythrocytes. A total of $10 \mu \mathrm{g}$ of PGA was added to $100 \mu \mathrm{L}$ of erythrocyte suspension, and then the mixtures were immediately supplemented with the cationic lipoplexes of $2 \mu \mathrm{g}$ of siRNA formed at a charge ratio (+/-) of $4 / 1$. After incubation for $15 \mathrm{~min}$ at $37^{\circ} \mathrm{C}$, the sample was placed on a glass plate and agglutination was observed by microscopy.

\section{Results and Discussion}

\subsection{Effect of Order of Administration of PGA and Cationic Lipoplex on Biodistribution of SiRNA}

Previously, we reported that sequential injection of PGA plus cationic lipoplex of siRNA could deliver siRNA into the liver [8]. In this study, we examined the effect of charge ratio (+/-) of cationic liposome/siRNA, molecular weight of PGA and cationic lipid of cationic liposome on the biodistribution of siRNA after sequential injection of PGA plus cationic lipoplex. We decided to use DOTAP/Chol liposomes as the cationic liposomes because they had often been used for in vivo applications for gene delivery [9] [10], and the lipoplex of pDNA encoding tumor suppressor gene TUSC2/FUS1 had been exploited in a clinical setting for the treatment of nonsmall cell lung cancer (NSCLC) patients [11]. DOTAP/Chol lipoplex was formed by mixing DOTAP/Chol liposome with siRNA at a charge ratio (+/-) of 4/1. In PGA, we used poly- $\alpha$-L-glutamic acid with a molecular weight of $20.5 \mathrm{kDa}$. With regard to the injected dose (mg) of PGA, $1 \mathrm{mg}$ was intravenously injected because we previously found that the sequential injection of this amount plus cationic lipoplex was associated with the highest accumulation of siRNA in the liver [8], comparable to those by commercially available transfection reagent Invivofectamine2.0 and hydrodynamic injection, which can efficiently introduce siRNA into the liver by intravenous injection.

First, we evaluated the effect of the order of administration of PGA and cationic lipoplex on the biodistribution of Cy5.5-siRNA in mice. When naked siRNA was injected, strong accumulation was observed only in the kidneys (Figure 2(a) and Figure 2(b)), indicating that naked siRNA was quickly eliminated from the body by filtration in the kidneys. When DOTAP/Chol lipoplex was injected, siRNA was largely accumulated in the lungs (Figure 2(a) and Figure 2(b)). This suggested that electrostatic interactions between positively charged DOTAP/Chol lipoplexes and negatively charged erythrocytes caused agglutination, and their agglutinates were entrapped in the highly extended lung capillaries. Upon the injection of DOTAP/Chol liposomes at $1 \mathrm{~min}$ after the injection of naked siRNA, siRNA was accumulated mainly in the lungs, but weakly in the liver (Figure 2(a) and Figure 2(b)), indicating that most of the siRNA interacted with DOTAP/Chol liposomes in blood circulation, and their complexes were entrapped in the lungs. This result is similar to previously reported findings that sequential injection of cationic liposome plus plasmid DNA effectively transfects the lung [12]. Upon the injection of cationic lipoplex at 1 min after the injection of PGA, strong accumulation of siRNA was observed in the liver and moderately in the kidneys (Figure 2(a) and Figure 2(b)), suggesting that PGA in blood circulation might prevent the agglutination of DOTAP/Chol lipoplex with erythrocytes, and increase the accumulation in the liver. We still have no evidence that intravenously injected PGA could interact with DOTAP/Chol lipoplex in blood circulation; however, we speculated that PGA injection might prevent the agglutination of DOTAP/ Chol lipoplex with erythrocytes by coating of the lipoplex with PGA in blood circulation, and increase the accumulation in the liver. However, some siRNAs might be dissociated from DOTAP/Chol lipoplexes in blood by PGA injection and be excreted by the kidneys.

In contrast, in the sequential injection of PGA at 1 min after the injection of DOTAP/Chol lipoplex, siRNA was accumulated mainly in the lungs (data not shown), suggesting that DOTAP/Chol lipoplex after intravenous injection might rapidly accumulate in the lungs before PGA injection. Moreover, upon the injection of DOTAP/ Chol liposomes at 1 min after the injection of a mixture of PGA and siRNA, marked accumulation of siRNA was observed in the kidney (Figure 2(a) and Figure 2(b)), indicating that individual injection of PGA, DOTAP/ Chol liposomes and siRNA could not form cationic lipoplex or ternary complex in the blood, and the most of the siRNA was rapidly eliminated from the kidneys. From these findings, the injection of PGA, followed by the injection of DOTAP/Chol lipoplex, was needed for siRNA delivery into the liver. 


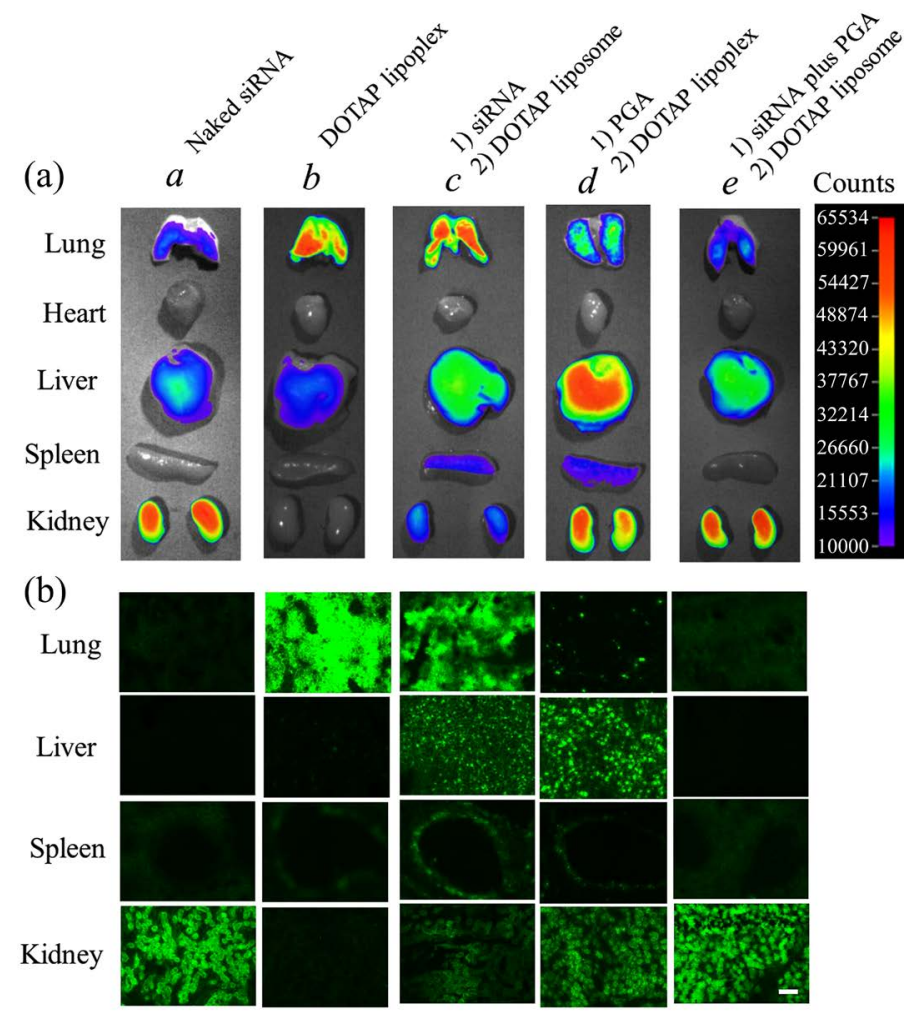

Figure 2. Ex vivo images of dissected tissues (a) and tissue section (b) at $1 \mathrm{~h}$ after the sequential injection of PGA plus DOTAP/Chol lipoplex into mice. DOTAP/Chol lipoplex was formed at a charge ratio $(+/-)$ of $4 / 1$. In $a$ and $b, 50 \mu \mathrm{g}$ of naked Cy5.5-siRNA and DOTAP/ Chol lipoplex of $50 \mu \mathrm{g}$ of Cy5.5-siRNA were injected into the mice. In $c$, DOTAP/Chol liposomes were injected at $1 \mathrm{~min}$ after the injection of $50 \mu \mathrm{g}$ of Cy5.5-siRNA. In $d$, DOTAP/Chol lipoplex of $50 \mu \mathrm{g}$ of Cy5.5-siRNA was injected at $1 \mathrm{~min}$ after the injection of $1 \mathrm{mg}$ of PGA. In $e$, DOTAP/Chol liposomes were injected at 1 min after the injection of a mixture of $50 \mu \mathrm{g}$ of Cy5.5-siRNA and $1 \mathrm{mg}$ of PGA. In (a), the exposure time for the detection of Cy5.5 fluorescence was 5 seconds. Fluorescence intensity is illustrated by a color-coded scale (red is maximum, purple is minimum). In (b), green signals indicate the localization of Cy5.5-siRNA. Scale bar $=100 \mu \mathrm{m}$.

\subsection{Effect of Charge Ratio (+/-) of Cationic Lipoplex on the Biodistribution of siRNA}

To investigate the effect of the charge ratio (+/-) of DOTAP/Chol liposomes and siRNA on the biodistribution after sequential injection of PGA plus DOTAP/Chol lipoplex, we intravenously injected DOTAP/Chol lipoplexes of Cy5.5-siRNA at charge ratios (+/-) of 6/1 and 8/1, after the injection of PGA into mice. The injection of DOTAP/Chol lipoplex at a charge ratio (+/-) of 6/1 or 8/1 after the injection of PGA exhibited high accumulation of siRNA in the liver (Figure 3(a) and Figure 3(b)), comparable to that at a charge ratio (+/-) of 4/1 (Figure 2(a) and Figure 2(b)). Although we speculated that an increased charge ratio $(+/-)$ could reduce the exclusion of siRNA from the kidneys due to high association between cationic liposomes and siRNA via electrostatic interaction, siRNAs were detected in the kidneys. As a result, the charge ratio (+/-) did not largely affect the biodistribution of siRNA after the sequential injection of PGA plus DOTAP/Chol lipoplex.

\subsection{Effect of Molecular Weight of PGA on Biodistribution of SiRNA}

Sequential injection of poly- $\alpha$-L-glutamic acid of $20.5 \mathrm{kDa}$ plus cationic lipoplex could deliver siRNA mainly in the liver (Figure 2(a) and Figure 2(b)). Next, we examined whether the molecular weight and isomer of PGA 
(a)

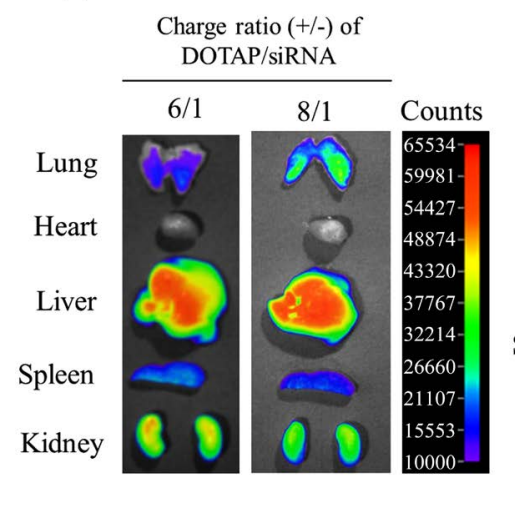

(b)

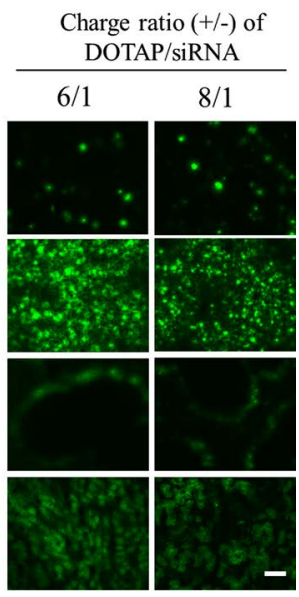

Figure 3. Effect of the charge ratio (+/-) of DOTAP/Chol lipoplex on the biodistribution of siRNA in mice after sequential injection of PGA plus DOTAP/Chol lipoplex of Cy5.5-siRNA. DOTAP/Chol lipoplexes were formed at charge ratios (+/-) of $6 / 1$ and $8 / 1$, and $e x$ vivo images of dissected tissues (a) and tissue section (b) were obtained at $1 \mathrm{~h}$ after the sequential injection of $1 \mathrm{mg}$ of PGA (20.5 kDa) plus DOTAP/Chol lipoplex of $50 \mu \mathrm{g}$ of Cy5.5-siRNA into mice. In (a), the exposure time for the detection of Cy5.5 fluorescence was 5 seconds. Fluorescence intensity is illustrated by a color-coded scale (red is maximum, purple is minimum). In (b), green signals indicate the localization of Cy5.5-siRNA. Scale bar $=100 \mu \mathrm{m}$.

affected the biodistribution of siRNA after the sequential injection of PGA plus DOTAP/Chol lipoplex (Figure 4(a) and Figure 4(b)). In the sequential injection of poly- $\alpha$-L-glutamic acid of $64 \mathrm{kDa}$, poly- $\alpha$-D-glutamic acid of $38 \mathrm{kDa}$ or poly- $\gamma$-L-glutamic acid of $200 \mathrm{kDa}$ plus cationic lipoplex, siRNA was detected mainly in the liver (Figure 4(b)). However, the sequential injection of poly- $\gamma$-L-glutamic acid of $200 \mathrm{kDa}$ plus DOTAP/Chol lipoplex exhibited lower accumulation of siRNA in the liver than those by other types of PGA (Figure 4(a)). This might indicate that large PGA ( 200 kDa) could not strongly interact with DOTAP/Chol lipoplex in the blood.

\subsection{Effect of Cationic Lipid of Cationic Lipoplex on Biodistribution of siRNA}

Next, we examined whether cationic lipid of cationic liposome affected the biodistribution of siRNA after the sequential injection of PGA (20.5 kDa) plus cationic lipoplex. We prepared DOTMA/Chol, DDAB/Chol and DC-6-14/Chol liposomes as cationic liposomes because DOTMA, DDAB and DC-6-14 have often been used as cationic lipids in liposomal formulations for gene delivery [13]-[15]. When DOTMA lipoplex was injected into mice, siRNA was largely accumulated in the lung and liver (Figure 5(a)). In contrast, when DDAB/Chol and DC-6-14/Chol lipoplexes were injected, the accumulation of siRNA was observed largely in the lung. However, pre-injection of PGA could decrease the accumulation of siRNA in the lung by the injection of DOTMA/Chol, DDAB/Chol or DC-6-14/Chol lipoplex, and increased it in the liver (Figure 5(a)). Among the cationic liposomes, DOTAP/Chol liposomes efficiently delivered siRNA in the liver after sequential injection (Figure 2 and Figure 5(a)).

Previously, we reported that the addition of PGA into erythrocyte suspension could prevent agglutination by DOTAP/Chol lipoplex [8]. Therefore, we evaluated the effect of agglutination with erythrocytes by DOTMA/ Chol, DDAB/Chol and DC-6-14/Chol lipoplexes after the treatment of erythrocytes with PGA. In terms of the results, the addition of PGA into erythrocyte suspension could prevent agglutination induced by the addition of their lipoplexes (Figure 5(b)), indicating that PGA might interact with their lipoplexes in the blood and decrease accumulation in the lungs by preventing association with blood components.

PEGylation on the surface of cationic lipoplex (PEG-modified lipoplex) can also decrease accumulation in the lungs by preventing association with blood components; however, PEGylation abolishes the effect of gene suppression by siRNA owing to the high stability of the lipoplex. Electrostatic encapsulation of cationic lipoplex 


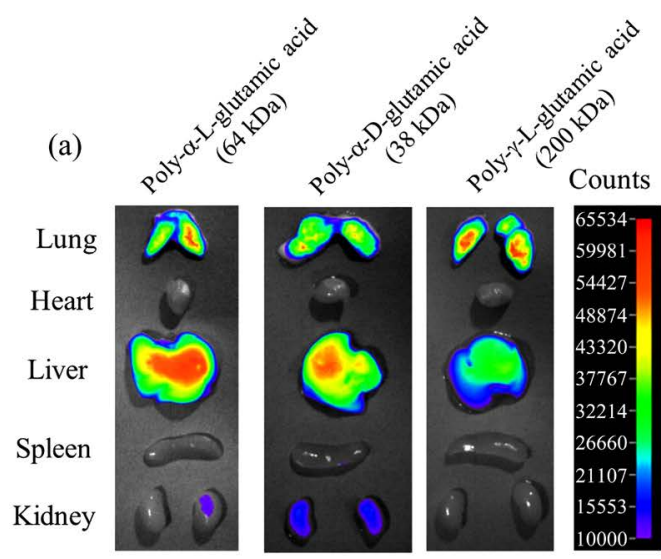

(b)

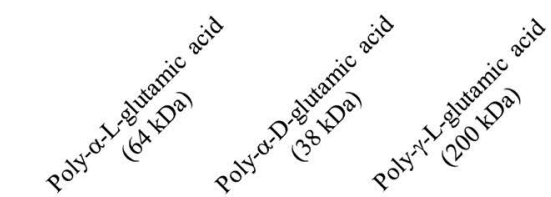

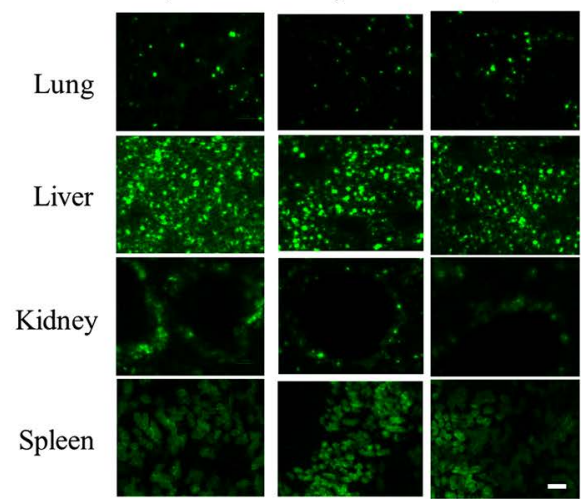

Figure 4. Effect of molecular weight of PGA on the biodistribution of siRNA in mice after the sequential injection of PGA plus DOTAP/Chol lipoplex. Ex vivo images of dissected tissues (a) and tissue section (b) were obtained at $1 \mathrm{~h}$ after sequential injection of $1 \mathrm{mg}$ of PGA plus DOTAP/Chol lipoplex of $50 \mu \mathrm{g}$ of Cy5.5-siRNA into mice. Poly- $\alpha$-L-glutamic acid of 64 $\mathrm{kDa}$, poly- $\alpha$-D-glutamic acid of $38 \mathrm{kDa}$ and poly- $\gamma$-L-glutamic acid of $200 \mathrm{kDa}$ were used as a PGA. In (a), the exposure time for the detection of Cy5.5 fluorescence was 5 seconds. Fluorescence intensity is illustrated by a color-coded scale (red is maximum, purple is minimum). In (b), green signals indicate the localization of Cy5.5-siRNA. Scale bar $=100 \mu \mathrm{m}$.

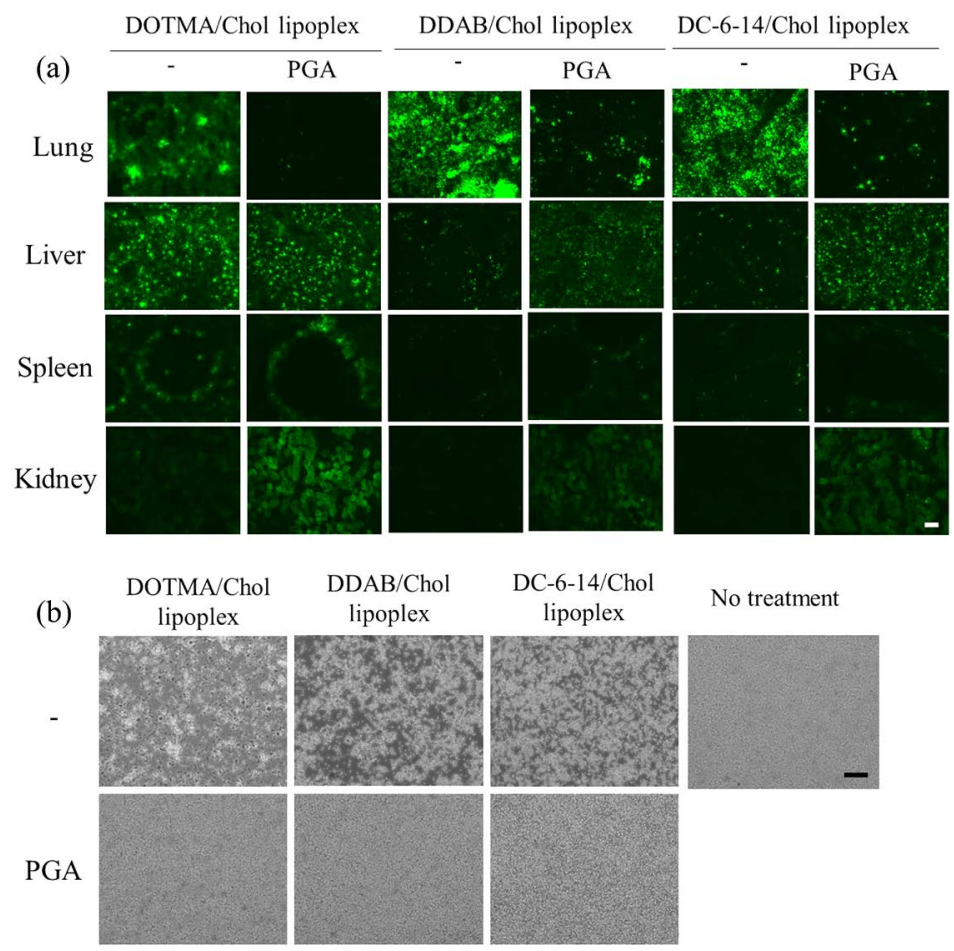

Figure 5. Effect of cationic liposomes on the biodistribution of siRNA in mice after sequential injection of PGA plus cationic lipoplex. DOTMA/Chol, DDAB/Chol and DC-6-14/Chol liposomes were used as cationic liposomes. Their cationic lipoplexes were formed by mixing with siRNA at a charge ratio (+/-) of 4/1. In (a), image of tissue section was obtained at 1 $\mathrm{h}$ after the sequential injection of $1 \mathrm{mg}$ of PGA (20.5 kDa) plus cationic lipoplex of $50 \mu \mathrm{g}$ of Cy5.5-siRNA into mice. Green signals indicate the localization of Cy5.5-siRNA. Scale bar $=100 \mu \mathrm{m}$. In (b), the agglutination of cationic lipoplexes with erythrocytes in the presence or absence of PGA (20.5 kDa) is shown. Immediately after the mixing of $10 \mu \mathrm{g}$ of PGA with erythrocytes, cationic lipoplex of $2 \mu \mathrm{g}$ of siRNA was added to them, and agglutination was observed by phase contrast microscopy. Scale bar $=100 \mu \mathrm{m}$. 
with anionic biodegradable polymers such as chondroitin sulfate and PGA can also prevent the agglutination with blood components [7] [16] [17]. However, in the coating of cationic lipoplex with anionic polymer (ternary complex), the size and $\zeta$-potential of the lipoplex were markedly affected by changing the charge ratio (+/-) of cationic lipoplex to anionic polymer [7]; therefore, this charge ratio $(+/-)$ must be precisely controlled for the preparation of anionic polymer-coating lipoplex before intravenous injection. In contrast, sequential injection has an advantage in that cationic lipoplex can be delivered into the liver without directly coating the lipoplex with anionic polymer before injection.

Resident macrophages in the liver called Kupffer cells comprise the major population of the reticuloendothelial system (RES). Intravenously injected liposomes were shown to be generally taken up by RES [18]. Therefore, there was a possibility that most of the cationic lipoplexes accumulated in the liver might be captured by Kupffer cells. Intravenous injections of bisphosphonate such as clodronic acid or zoledronic acid (ZOL) can significantly deplete macrophages in the liver of mice [19]. Therefore, we sequentially injected PGA plus DOTAP/Chol lipoplex into the mice treated with ZOL. However, the decrease of macrophages in the liver by ZOL did not affect the biodistribution of siRNA after the sequential injection of PGA plus DOTAP/Chol lipoplex (data not shown). These findings indicated that siRNA after the sequential injection of PGA plus cationic lipoplex might not be accumulated in the liver through the uptake by Kupffer cells. However, we have no information about regions in which siRNA was localized in the liver after the sequential injection of PGA plus cationic lipoplex, but most of the siRNA in the liver was co-localized with DOTAP/Chol liposomes there (data not shown).

Previously, we reported that the sequential injection of PGA plus DOTAP/Chol lipoplex of cholesterol-conjugated ApoB siRNA could induce suppression of the ApoB mRNA level in the liver [8]. However, in unconjugated siRNA, sequential injection of PGA plus DOTAP/Chol lipoplex of ApoB siRNA did not reduce ApoB expression in the liver (unpublished data). It has also been reported that lipid conjugates of siRNAs enhance cellular uptake and gene silencing in liver cells [20]. In hepatocyte targeting by sequential injection, lipid modification of siRNA might facilitate the uptake of siRNA into hepatocytes and be able to induce a gene silencing effect. Furthermore, modification of siRNA with $N$-acetylgalactosamine ligands that target asialoglycoprotein receptors on hepatocytes is also used for targeting to hepatocytes [21]. Further study must be performed to investigate the optimal siRNA modification for the gene silencing effect in the liver after sequential injection.

\section{Conclusion}

In this study, we found that sequential injection of PGA plus cationic liposomes could deliver siRNA into the liver, but the charge ratio (+/-) of cationic lipoplex, molecular weight of PGA and cationic lipid of cationic liposomes did not largely affect the biodistribution of siRNA after sequential injection. Sequential injection of PGA and cationic lipoplex might produce a systemic vector of siRNA to the liver.

\section{Acknowledgements}

This project was supported in part by a Grant-in-Aid for Scientific Research (C) from the Japan Society for the Promotion of Science (KAKENHI Grant Number 26460046).

\section{References}

[1] Kubowicz, P., Zelaszczyk, D. and Pekala, E. (2013) RNAi in Clinical Studies. Current Medicinal Chemistry, 20, 1801-1816. http://dx.doi.org/10.2174/09298673113209990118

[2] Zhang, S.B., Zhi, D.F. and Huang, L. (2012) Lipid-Based Vectors for siRNA Delivery. Journal of Drug Targeting, 20, 724-735. http://dx.doi.org/10.3109/1061186X.2012.719232

[3] Zhou, J.H., Shum, K.T., Burnett, J.C. and Rossi, J.J. (2013) Nanoparticle-Based Delivery of RNAi Therapeutics: Progress and Challenges. Pharmaceuticals, 6, 85-107. http://dx.doi.org/10.3390/ph6010085

[4] de Fougerolles, A.R. (2008) Delivery Vehicles for Small Interfering RNA in Vivo. Human Gene Therapy, 19, $125-132$. http://dx.doi.org/10.1089/hum.2008.928

[5] Eliyahu, H., Servel, N., Domb, A.J. and Barenholz, Y. (2002) Lipoplex-Induced Hemagglutination: Potential Involvement in Intravenous Gene Delivery. Gene Therapy, 9, 850-858.

[6] Simberg, D., Weisman, S., Talmon, Y., Faerman, A., Shoshani, T. and Barenholz, Y. (2003) The Role of Organ Vas- 
cularization and Lipoplex-Serum Initial Contact in Intravenous Murine Lipofection. The Journal of Biological Chemistry, 278, 39858-39865. http://dx.doi.org/10.1074/jbc.M302232200

[7] Hattori, Y., Nakamura, A., Arai, S., Nishigaki, M., Ohkura, H., Kawano, K., Maitani, Y. and Yonemochi, E. (2014) In Vivo siRNA Delivery System for Targeting to the Liver by Poly-L-Glutamic Acid-Coated Lipoplex. Results in Pharma Sciences, 4, 1-7. http://dx.doi.org/10.1016/j.rinphs.2014.01.001

[8] Hattori, Y., Arai, S., Okamoto, R., Hamada, M., Kawano, K. and Yonemochi, E. (2014) Sequential Intravenous Injection of Anionic Polymer and Cationic Lipoplex of siRNA Could Effectively Deliver siRNA to the Liver. International Journal of Pharmaceutics, 476, 289-298. http://dx.doi.org/10.1016/j.ijpharm.2014.09.059

[9] Song, Y.K., Liu, F., Chu, S.Y. and Liu, D.X. (1997) Characterization of Cationic Liposome-Mediated Gene Transfer in Vivo by Intravenous Administration. Human Gene Therapy, 8, 1585-1594. http://dx.doi.org/10.1089/hum.1997.8.13-1585

[10] Templeton, N.S., Lasic, D.D., Frederik, P.M., Strey, H.H., Roberts, D.D. and Pavlakis, G.N. (1997) Improved DNA: Liposome Complexes for Increased Systemic Delivery and Gene Expression. Nature Biotechnology, 15, 647-652. http://dx.doi.org/10.1038/nbt0797-647

[11] Lu, C., Stewart, D.J., Lee, J.J., Ji, L., Ramesh, R., Jayachandran, G., Nunez, M.I., Wistuba, I.I., Erasmus, J.J., Hicks, M.E., Grimm, E.A., Reuben, J.M., Baladandayuthapani, V., Templeton, N.S., McMannis, J.D. and Roth, J.A. (2012) Phase I Clinical Trial of Systemically Administered TUSC2(FUS1)-Nanoparticles Mediating Functional Gene Transfer in Humans. PloS ONE, 7, e34833. http://dx.doi.org/10.1371/journal.pone.0034833

[12] Tan, Y., Liu, F., Li, Z., Li, S. and Huang, L. (2001) Sequential Injection of Cationic Liposome and Plasmid DNA Effectively Transfects the Lung with Minimal Inflammatory Toxicity. Molecular Therapy, 3, 673-682. http://dx.doi.org/10.1006/mthe.2001.0311

[13] Tagami, T., Suzuki, T., Matsunaga, M., Nakamura, K., Moriyoshi, N., Ishida, T. and Kiwada, H. (2012) Anti-Angiogenic Therapy via Cationic Liposome-Mediated Systemic siRNA Delivery. International Journal of Pharmaceutics, 422, 280-289. http://dx.doi.org/10.1016/j.ijpharm.2011.10.059

[14] Wollenberg, B., Kastenbauer, H.M., Schaumberg, J., Mayer, A., Andratschke, M., Lang, S., Pauli, C., Zeidler, R., Ihrler, S., Lohrs, K.N. and Rollston, R. (1999) Gene Therapy—Phase I Trial for Primary Untreated Head and Neck Squamous Cell Cancer (HNSCC) UICC Stage II-IV with a Single Intratumoral Injection of hIL-2 Plasmids Formulated in DOTMA/Chol. Human Gene Therapy, 10, 141-147. http://dx.doi.org/10.1089/10430349950019273

[15] Jin, Y., Wang, S., Tong, L. and Du, L. (2015) Rational Design of Didodecyldimethylammonium Bromide-Based Nanoassemblies for Gene Delivery. Colloids and Surfaces B: Biointerfaces, 126, 257-264. http://dx.doi.org/10.1016/j.colsurfb.2014.12.032

[16] Kurosaki, T., Kitahara, T., Fumoto, S., Nishida, K., Yamamoto, K., Nakagawa, H., Kodama, Y., Higuchi, N., Nakamura, T. and Sasaki, H. (2010) Chondroitin Sulfate Capsule System for Efficient and Secure Gene Delivery. Journal of Pharmacy \& Pharmaceutical Sciences, 13, 351-361.

[17] Kurosaki, T., Kitahara, T., Kawakami, S., Higuchi, Y., Yamaguchi, A., Nakagawa, H., Kodama, Y., Hamamoto, T., Hashida, M. and Sasaki, H. (2010) Gamma-Polyglutamic Acid-Coated Vectors for Effective and Safe Gene Therapy. Journal of Controlled Release, 142, 404-410. http://dx.doi.org/10.1016/j.jconrel.2009.11.010

[18] Moghimi, S.M. and Hunter, A.C. (2001) Recognition by Macrophages and Liver Cells of Opsonized Phospholipid Vesicles and Phospholipid Headgroups. Pharmaceutical Research, 18, 1-8. http://dx.doi.org/10.1023/A:1011054123304

[19] Hattori, Y., Yamashita, J., Sakaida, C., Kawano, K. and Yonemochi, E. (2015) Evaluation of Antitumor Effect of Zoledronic Acid Entrapped in Folate-Linked Liposome for Targeting to Tumor-Associated Macrophages. Journal of Liposome Research, in Press.

[20] Lorenz, C., Hadwiger, P., John, M., Vornlocher, H.P. and Unverzagt, C. (2004) Steroid and Lipid Conjugates of siRNAs to Enhance Cellular Uptake and Gene Silencing in Liver Cells. Bioorganic \& Medicinal Chemistry Letters, 14, 4975-4977. http://dx.doi.org/10.1016/j.bmcl.2004.07.018

[21] Nair, J.K., Willoughby, J.L., Chan, A., Charisse, K., Alam, M.R., Wang, Q., Hoekstra, M., Kandasamy, P., Kel’in, A.V., Milstein, S., Taneja, N., O’Shea, J., Shaikh, S., Zhang, L., van der Sluis, R.J., Jung, M.E., Akinc, A., Hutabarat, R., Kuchimanchi, S., Fitzgerald, K., Zimmermann, T., van Berkel, T.J., Maier, M.A., Rajeev, K.G. and Manoharan, M. (2014) Multivalent N-Acetylgalactosamine-Conjugated siRNA Localizes in Hepatocytes and Elicits Robust RNAiMediated Gene Silencing. Journal of the American Chemical Society, 136, 16958-16961. http://dx.doi.org/10.1021/ja505986a 